\title{
Validation Test of a Cam Mover Based Micrometric Pre-Alignment System for Future Accelerator Components
}

\author{
J. Kemppinen, F. Lackner, H. Mainaud Durand \\ European Organisation for Nuclear Research, Geneva, Switzerland, Juha.Kemppinen@cern.ch
}

\begin{abstract}
Compact Linear Collider (CLIC) is a $48 \mathrm{~km}$ long linear accelerator currently studied at CERN. It is a high luminosity electronpositron collider with an energy range of $0.5-3 \mathrm{TeV}$. CLIC is based on a two-beam technology in which a high current drive beam transfers RF power to the main beam accelerating structures. The main beam is steered with quadrupole magnets. Main beam components have to be actively pre-aligned within $14 \mu \mathrm{m}$ in sliding windows of $200 \mathrm{~m}$. To reach the pre-alignment requirement as well as the rigidity required by nano-stabilization, a system based on eccentric cam movers is proposed for the re-adjustment of the main beam quadrupoles. Validation of the technique to the stringent CLIC requirements was started with tests in one degree of freedom on an eccentric cam mover. This paper describes the dedicated mock-up as well as the tests and measurements carried out with it. Finally, the test results are presented.
\end{abstract}

Keywords: CLIC, main beam quadrupole, eccentric cam mover, alignment

\section{INTRODUCTION}

$\mathrm{T}$ HE CLIC MAIN BEAM (MB) will be steered using approximately 4000 quadrupole magnets which are divided into four types. The quadrupole length varies between $420 \mathrm{~mm}$ and $1915 \mathrm{~mm}$ depending on the type. To reach the design luminosity $2.3-5.9 \cdot 10^{34} \mathrm{~cm}^{-2} \mathrm{~s}^{-1}$, the CLIC main beam has to be stabilized to $1 \mathrm{~nm}$ in vertical and $40 \mathrm{~nm}$ in horizontal direction above $1 \mathrm{~Hz}$ [1]. This will be performed by a nano-stabilization system which requires high stiffness from the re-adjustment system on top of which it will be installed. In order to implement beam based alignment and beam based feedback, the CLIC main beam will have to be pre-aligned within $14 \mu \mathrm{m}$ rms in sliding windows of $200 \mathrm{~m}$. This means that the MB quadrupole magnets and their associated beam position monitors (BPM) will have to be re-adjusted with a precision and accuracy of approximately $1 \mu \mathrm{m}$ in 5 degrees of freedom (d.o.f.).

A system based on eccentric cam movers was chosen for the CLIC MB quadrupole re-adjustment. Cam mover based alignment systems are already in use in several accelerators and synchrotrons but with less stringent requirements. Cam movers provide both good resolution and high stiffness [2].

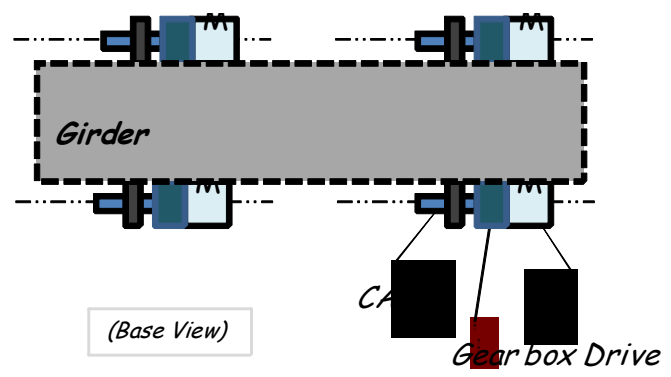

Fig.1. Cam based alignment concept in 5 d.o.f

The re-adjustment system consists of 5 cam movers which are used to manipulate a quadrupole magnet in 5 d.o.f. It is a 3 point support contact with 4 interfaces with respect to the basement. The basic concept of alignment in 5 d.o.f. based on cam movers is shown in a base view sketch in Fig.1.

\section{ECCENTRIC CAM MOVER}

CLIC sets very stringent pre-alignment requirements to its MB quadrupoles. The re-adjustment systems that realize the pre-alignment have to be capable of extremely precise and high resolution movement. At the same time, the readjustment systems have to provide stiffness so that the nano-stabilization systems can meet their requirements.

Several re-adjustment methods were considered for the active pre-alignment of CLIC MB quadrupoles. Hexapod structures were excluded due to their lower rigidity. Wedge systems were considered but had too much contact friction in order to reach the micrometric re-positioning requirement. The most promising method was based on eccentric cam movers and it was chosen for further studies.

A cam mover is an actuator which converts rotation to translation through an eccentric cam shaft. Fig.2 is a drawing of an eccentric cam shaft.

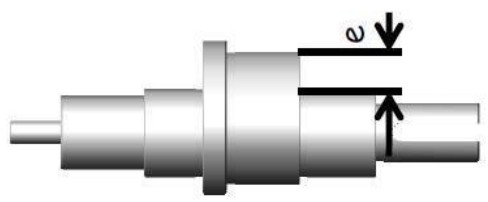

Fig.2. Eccentric cam shaft.

The conversion from rotation to translation is not linear but follows a sinusoidal curve. The stroke of a cam mover is determined by the eccentricity (see "e" in Fig.2). The full stroke is $2 \mathrm{e}$ and it is achieved by rotating the cam shaft by 180 degrees.

Cam movers were originally developed for the positioning of beamline magnets of the Final Focus Test Beam at Stanford Linear Accelerator Center (SLAC). [3] Since then, they have been used successfully in the alignment of several accelerators and synchrotrons. For example, Paul Scherrer Institute (PSI) used them in the alignment of the Swiss Light Source (SLS) [4]. Their design was used as a baseline concept within the CLIC study. However, it was required to determine various parameters and to further develop the 
system's performance. The focus was on optimizing the cam diameter and its thickness in order to obtain the maximum stiffness. The Hertz theory was used for this optimization. One can see from Fig. 3 that the stiffness increases when increasing the diameter while reducing the deformation in the contact region. This optimization is therefore leading to higher Eigenfrequency which is required for the implementation of the nm stabilization equipment.

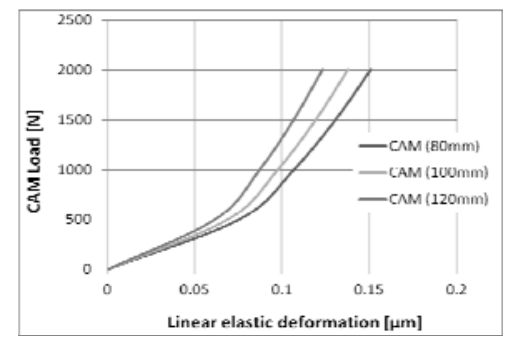

Fig.3. Linear elastic deformation vs. load and cam diameter variation.

Fig. 4 shows the relation of the cam radius and the Hertzian stress for the chosen cam diameter of $120 \mathrm{~mm}$. The stress increases when decreasing the cam diameter.

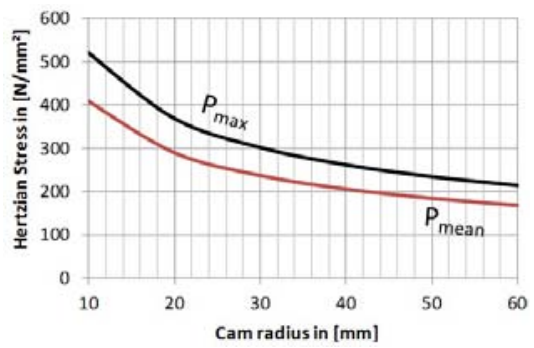

Fig.4. Linear elastic deformation vs. load and cam diameter variation.

Fig. 5 is a photo of a prototype cam mover. The eccentric cam shaft is mounted inside a chassis (a). A combination of a bearing and a bearing housing is mounted around the cam shaft (b). The housing acts as the mechanical interface with the cam follower.

The cam mover prototype's powertrain consists of commercial, off-the-shelf (COTS) products. The cam shaft is rotated by a standard 2-pole Oriental Motor stepper motor through a 5:1 worm drive and a 144:1 planetary gearbox. Both gearboxes are made by Gysin. The stepper motor is controlled using a motor drive from Pacific Scientific and a stepper motor controller from National Instruments. The controller has micro-stepping capability.

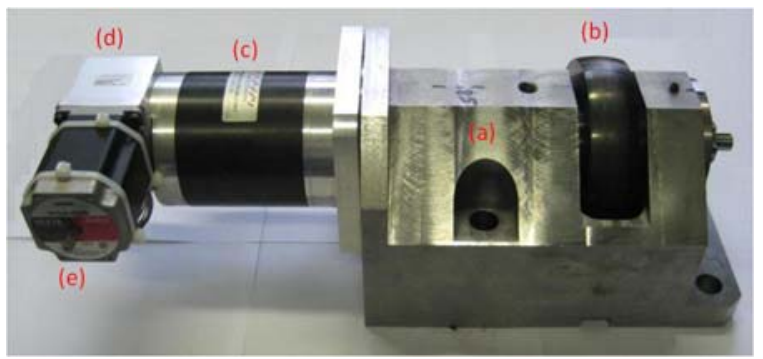

Fig.5 . Eccentric cam mover

\section{ONE DEGREE OF FREEDOM TESTS}

Validation of the CLIC MB quadrupole re-adjustment system based on eccentric cam movers was started with tests in 1 d.o.f. The cam mover geometry used in the first tests is based on the design used at the SLS [4]. The entire assembly was further developed by minimizing clearances and implementing state of the art components (gearbox etc.). This section will present the 1 d.o.f. mock-up and the repeatability tests carried out using it.

\section{A. One degree of freedom mock-up}

Fig.6 presents the 1 d.o.f. mock-up described in [5]. The cam mover (A) is installed under a cam follower plate (B). The plate is attached to rigid walls with four low friction linear guidings $(\mathrm{C})$. The guidings limit the plate's motion to one degree of freedom (vertical in Fig.4). The plate (B) weighs $35 \mathrm{~kg}$. An additional weight of $100 \mathrm{~kg}$ is mounted on top of the plate.

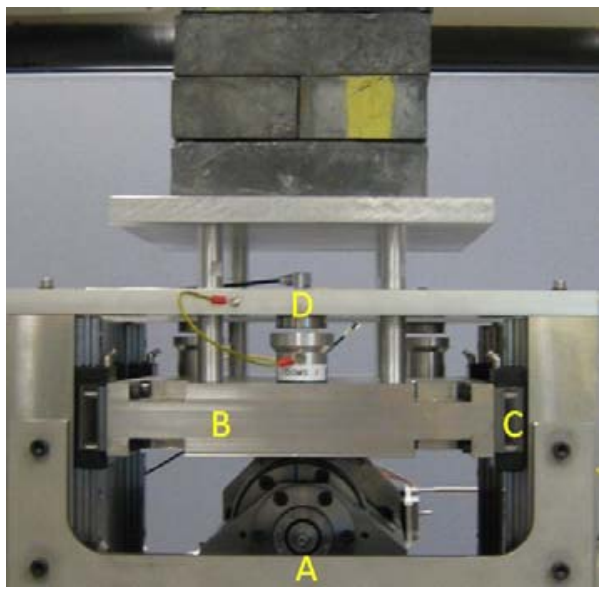

Fig.6. Side view of the one degree of freedom mock-up.

One can imagine that this setup follows the theory of a one mass oscillator (cf. Fig.7). The Hertzian contact with its linear elastic behavior is representing the spring of the system. The setup was designed to be interchangeable and to define the smallest repeatable alignment step for the individual cam and bearing combinations. The hardware was therefore implemented as a highly rigid system to minimize parasitic alignment effects due to elastic deformations in the setup. Finite element analysis (FEA) computations based on ANSYS software were performed to optimize the structure.

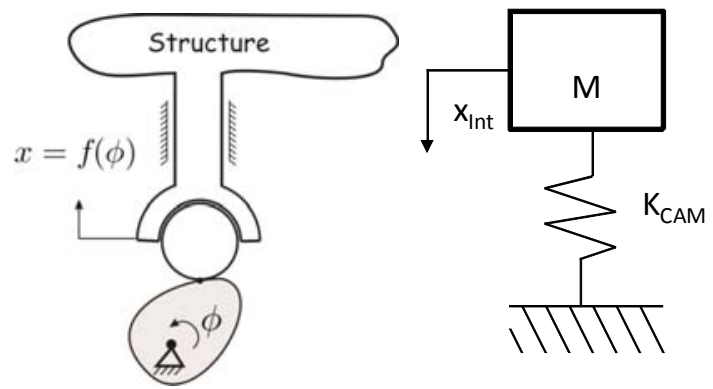

Fig.7. Cam system as single mass oscillator. 
A further purpose of the 1 d.o.f. mock-up is to test the repeatable plate position when rotating the cam mover by a complete revolution. All vertical displacement measurements are taken on the top plate position. Three capacitive Dimensional Offset Measurement Systems (DOMS) with resolution below $0.1 \mu \mathrm{m}$ were installed for this measurement [6]. The DOMS are shown as (D) in Fig.6.

Fig.8 shows the setup to measure the upper plate's $\mathrm{z}$ position. Three DOMS were used instead of one to have redundancy and to measure the inclination of the upper plate. The vertical position of the contact plane (CP in Fig.8) between the cam mover and the upper plate can be calculated based on the DOMS readings.

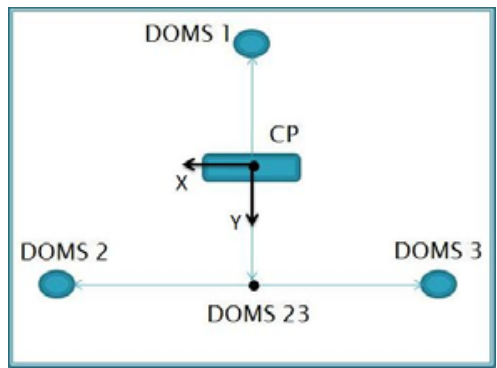

Fig.8. Top view of the DOMS sensors measuring the upper plate's z-position viewed from above. $\mathrm{CP}$ is the contact plane between the cam mover and the upper plate.

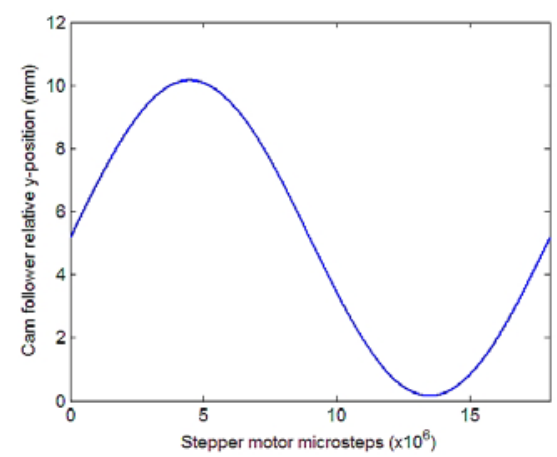

Fig.9. DOMS reading as a function of stepper motor microsteps measured in the 1 d.o.f. mock-up.

Fig.9 shows the readings of one of the DOMS when the cam shaft is rotated a full revolution. It can be seen in the figure that translation resolution depends on the orientation of the cam shaft. The worst case is in the mid-range where the sinusoidal curve is steepest.

The test setup is controlled by using a program created in the LabVIEW environment which is described in [7].

\section{B. Test Methods}

A cylindrical enclosure (housing) acts as the mechanical interface between the cam mover and the cam follower. In the case of the 1 d.o.f. mock-up, the upper plate (B in Fig.6) acts as the cam follower. Linear movement of the housing is realized by rotating an eccentric cam shaft which is mounted inside the housing. A bearing is installed between the cam shaft and the housing.

Two different types of bearings were tested; spherical roller bearing and cylindrical roller follower. In addition, two types of housings were tested; again with cylindrical and spherical outer surfaces. Both housings have induction hardened surfaces. Three different configurations were built using these bearing and housing types and tested in the 1 d.o.f. mock-up.

Table 1. Configurations tested in the 1 d.o.f. mock-up

\begin{tabular}{|c|c|c|c|}
\hline $\begin{array}{c}\text { Config. } \\
\text { No. }\end{array}$ & Bearing type & $\begin{array}{c}\text { Bearing } \\
\text { reference }\end{array}$ & $\begin{array}{c}\text { Housing } \\
\text { type }\end{array}$ \\
\hline 1 & $\begin{array}{c}\text { Spherical } \\
\text { roller bearing }\end{array}$ & SKF 22209 E & Cylindrical \\
\hline 2 & $\begin{array}{c}\text { Roller } \\
\text { follower }\end{array}$ & $\begin{array}{c}\text { IKO NAST } \\
45 \text { ZZUU }\end{array}$ & Cylindrical \\
\hline 3 & $\begin{array}{c}\text { Roller } \\
\text { follower }\end{array}$ & $\begin{array}{c}\text { IKO NAST } \\
45 \text { ZZUUR }\end{array}$ & Spherical \\
\hline
\end{tabular}

Table1 is showing the three tested configurations. Bearing references are listed in the table in addition to bearing and housing types. Configuration 1 consists of a spherical roller bearing and a cylindrical housing. Configurations 2 and 3 both have a roller follower bearing. The only difference between the bearings used in configurations 2 and 3 is the outer ring. It is cylindrical in configuration 2 and crowned in configuration 3 . The housing is cylindrical in configuration 2 and spherical in configuration 3 .

For each configuration, preparatory tests were carried out to find the best parameters for the repeatability test. The optimized parameters were: the number of data acquisitions after each sequence, the number of test drives in a test series, the settling time after a sequence and before data acquisition and the stepper motor reference speed. The cam mover has a traditional trapezoidal motion profile. The stepper motor reference speed means the constant maximum speed in the trapezoid.

A test series was performed based on the optimized parameters to find out the cam mover repeatability within the whole stroke of $10 \mathrm{~mm}$. In the beginning of the test, the cam follower plate was at its upper dead center. One test drive consisted of two parts. First, the plate was driven from the upper dead center to the lower dead center (PI revolution) and vice versa in the further iteration. The cam mover was stopped 20 times per PI revolution for measurements. This procedure was repeated 10 times. The mean values and root mean square errors (RMSE) were calculated between corresponding measurement points of all the 10 test drives of the test series.

The cam mover's range in vertical motion (stroke) is $10 \mathrm{~mm}$. This translates through the planetary gearbox and the worm drive to nine million micro steps of the stepper motor. The repeatability test series was repeated with a reduced stroke of the cam mover. One million micro steps were omitted from both ends of the stroke.

\section{RESULTS}

The results of the repeatability tests of configurations 1-3 are shown in Fig.10-12, respectively. In each figure, (a) presents the full stroke of nine million stepper motor micro steps and (b) presents the reduced stroke between 1 and 8 million micro steps from the upper dead center. 


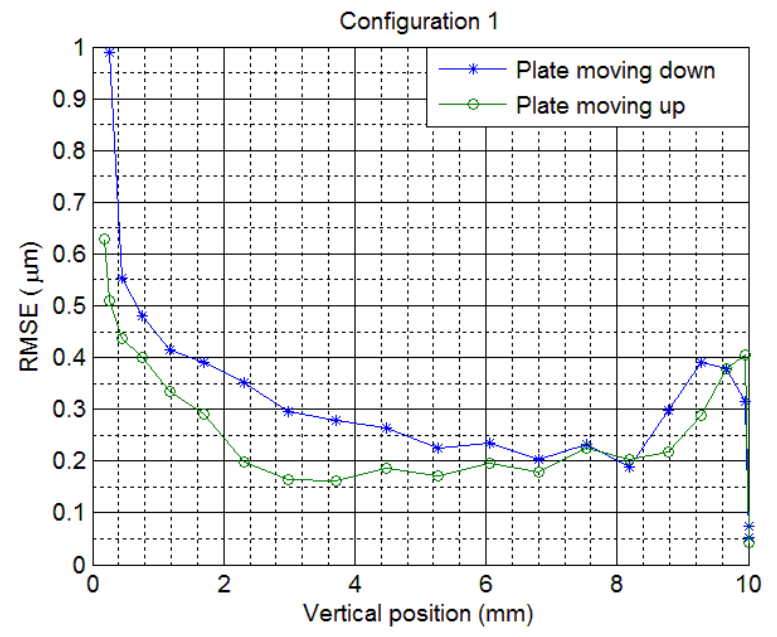

(a) Full stroke of 9 million stepper motor micro steps.

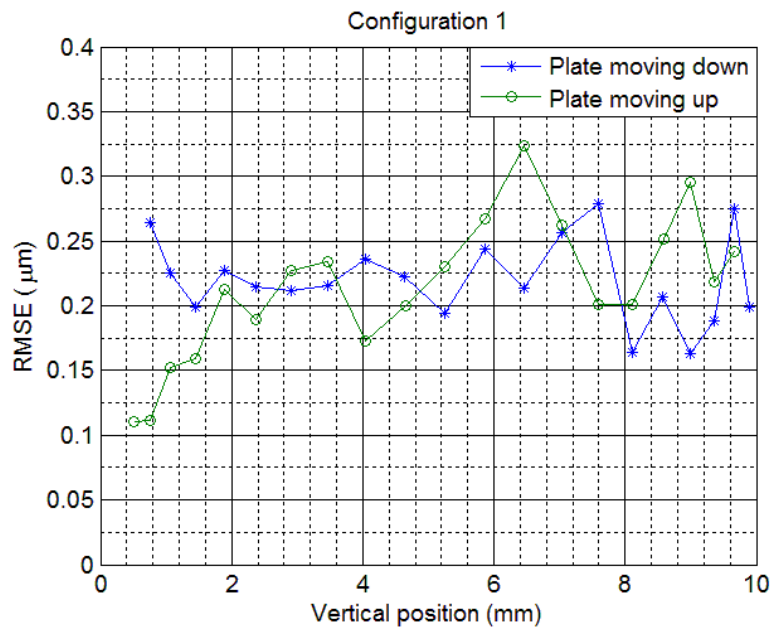

(b) Range between 1 and 8 million micro steps.

Fig.10. Repeatability as a function of measured vertical position of the cam follower plate using configuration 1 in the 1 d.o.f. mock-up.

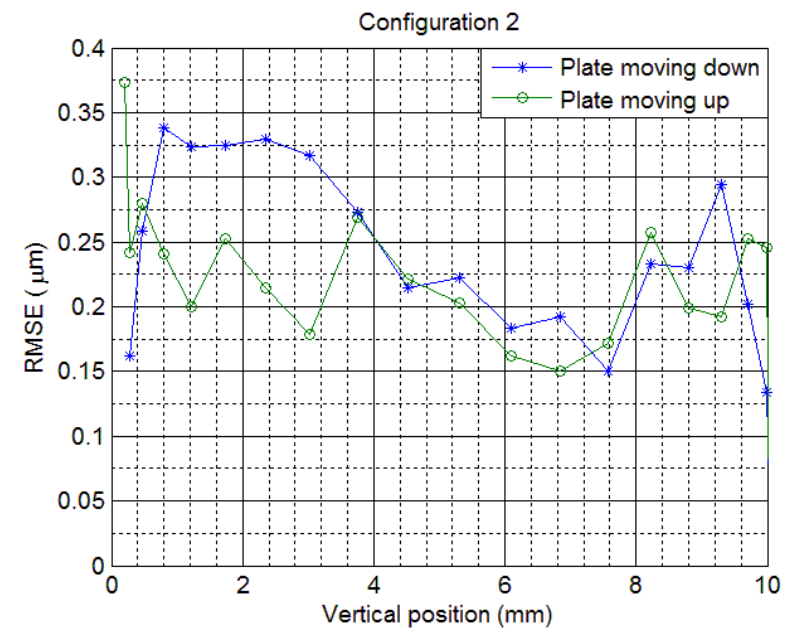

(a) Full stroke of 9 million stepper motor micro steps.

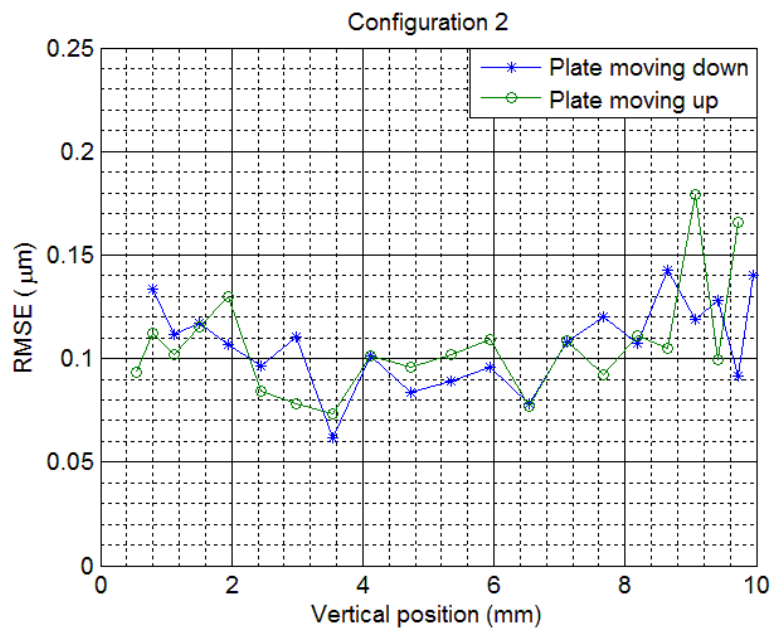

(b)..Range between 1 and 8 million micro steps.

Fig.11. Repeatability as a function of measured vertical position of the cam follower plate using configuration 2 in the 1 d.o.f. mock-up

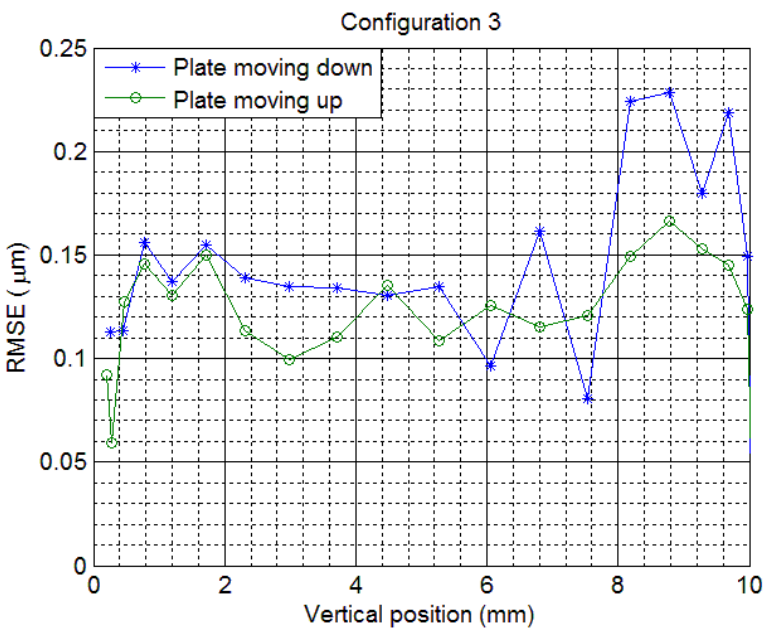

(a)..Full stroke of 9 million stepper motor micro steps.

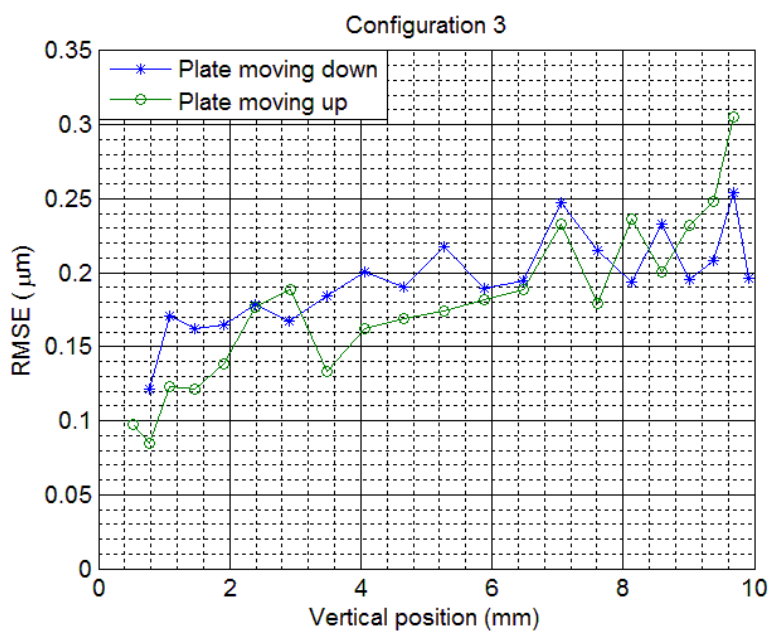

(b) Range between 1 and 8 million micro steps.

Fig.12. Repeatability as a function of measured vertical position of the cam follower plate using configuration 3 in the 1 d.o.f. mock-up. 
As can be seen in Fig.10-12, the repeatability was below micron with each configuration throughout the range. Configuration 3 had the best repeatability with full stroke. Configuration 2 had the best repeatability, below $0.2 \mu \mathrm{m}$, with reduced stroke.

Configurations 1 and 2 had better repeatability with the reduced stroke than with the full one. For configuration 3 , the results were better with full stroke than with partial stroke.

\section{DISCUSSION}

The 1 d.o.f. tests described in this article focused on the repeatability of cam mover movements. There is no calibration procedure defined in the setup so measuring precision or accuracy was not meaningful.

The 1 d.o.f. mock-up is controlled in open loop. Also, the effects of backlash and hysteresis of the powertrain were not taken into consideration in the reference commands which were sent to the cam mover. Therefore, the backlash and the hysteresis were included in the measurement results. In the case of the first CLIC cam mover prototype, the backlash and hysteresis were in the magnitude of several tens of microns. Thus, the precision requirements could not have been met without eliminating the backlash and the hysteresis.

In the repeatability tests, however, the effects of backlash and hysteresis are not seen, only their variation. And since the repeatability was all the time below micron, the backlash and the hysteresis are stable and can thus be eliminated fairly simply.

The next cam mover version will be equipped with a high end rotary absolute encoder. The encoder will be installed on the eccentric cam shaft so it will automatically eliminate the nonlinearities of the powertrain. It will also enable a simple calibration procedure for the cam movers.

Clearances inside the spherical roller bearing allow small lateral movement whereas the roller follower blocks it. The 1 d.o.f. mock-up diminishes efficiently movements in $\mathrm{x}$ - and $\mathrm{y}$-directions. Therefore, not all the effects of the clearances might have been visible in the 1 d.o.f. test results.

A 5 d.o.f. mock-up will be built for the validation of the sub-micrometric resolution cam mover system. The cam mover design has been further improved from the one used in the 1 d.o.f. mock-up. The structure is now more rigid, the clearances between parts are smaller, the worm drive and the stepper motor have been changed and there is a zero backlash bearing reducer instead of the planetary gearbox. Both bearing types will be tested also in 5 d.o.f.

In addition to positioning precision, the re-adjustment system has to provide a rigid support for the MB quadrupoles. The system's Eigenfrequency should be as high as possible. This has been taken into consideration in the cam mover design optimization process. The validation of the 5 d.o.f. concept will show if a further iteration in the re-adjustment system design will be required.

\section{CONCLUSION}

The CLIC main beam quadrupoles have to be prealigned within approximately $1 \mu \mathrm{m}$ in 5 d.o.f. A system based on eccentric cam movers is proposed for the readjustment. The system's validation process was started with repeatability tests in 1 d.o.f.

The cam mover was tested with three different bearing and housing configurations in 1 d.o.f. The repeatability of all of the three configurations was below $1.0 \mu \mathrm{m}$. The roller follower had better repeatability than the spherical roller bearing with both outer rings and housings. With reduced stroke, the differences between the configurations were negligible.

The good results from the 1 d.o.f. tests justify proceeding to the next phase in the validation of the MB quadrupole pre-alignment system based on eccentric cam movers.

\section{ACKNOWLEDGMENT}

The work done in Paul Scherrer Institute on cam movers has been very helpful in the CLIC baseline concept. Also Michel Rousseau and Sylvain Mico of CERN BE/ABP-SU section have given a lot of input in the work done.

\section{REFERENCES}

[1] Artoos, K., Capatina, O., Collette, C., Guinchard, M., Hauviller, C., Lackner, F., Pfingstner, J., Schmickler, H., Sylte, M. (2009). Study of the stabilization to the nanometer level of mechanical vibrations of the CLIC main beam quadrupoles. In Proceedings of the $23^{\text {rd }}$ Particle Accelerator Conference, 4-8 May 2009. Vancouver, Canada: Canada's National Laboratory for Particle and Nuclear Physics.

[2] Mainaud Durand, H., Touzé, T., Griffet, S., Kemppinen, J., Lackner, F. (2010). CLIC active pre-alignment system: Proposal for CDR and program for TDR. In Proceedings of the $11^{\text {th }}$ International Workshop on Accelerator Alignment, 13-17 September 2010. Hamburg, Germany: Deutsches Elektronen-Synchrotron DESY.

[3] Bowden, G., Holik, P., Wagner, S.R., Heimlinger, G., Settles, R. (1996). Precision magnet movers for the Final Focus Test Beam. Nuclear Instruments and Methods in Physics Research A, 368 (3), 579-592.

[4] Schlott, V., Kramert, R., Rohrer, M., Streun, A., Wiegand, P., Zelenika, S. (2000). Dynamic alignment at SLS. In Proceedins of the $7^{\text {th }}$ European Particle Accelerator Conference, 26-30 June 2000. Vienna, Austria: Institute of High Energy Physics of the Austrian Academy of Sciences, 693-695. 
[5] Lackner, F., Artoos, K., Collette, C., Mainaud Durant, H., Hauviller, C., Kemppinen, J., Leuxe, R. (2010). Development of an eccentric CAM based active pre-alignment system for the CLIC main beam quadrupole magnet. In Proceedings of the $6^{\text {th }}$ International Conference on Mechanical Engineering Design of Synchrotron Radiation Equipment and Instrumentation, 11-14 July 2010. Oxford, UK: Diamond Light Source.

[6] Herty, A. (2009). Micron precision calibration methods for alignment sensors in particle accelerators. Master's Thesis. Nottingham Trent University, Nottingham, UK.
[7] Otomański, P., Szlachta, A. (2008). The evaluation of expanded uncertainty of measurement results in direct measurements using the LabVIEW environment. Measurement Science Review, 8 (6), 147-150.
Received March 16, 2012. Accepted September 14, 2012. 